\title{
Exploring the antecedents, drivers, and outcome of Behaviour-based safety: A literature review
}

\author{
Sujata Sinha ${ }^{1}$, Dr.Ashutosh Muduli \\ ${ }^{1,2}$ School of Petroleum Management, Pandit Deendayal Petroleum University \\ ${ }^{1}$ sujata.sphd19@spm.pdpu.ac.in, ${ }^{2}$ ashutosh.Muduli@spm.pdpu.ac.in
}

\begin{abstract}
Organisations good at managing safety tend to manage operations well. Safety culture is a subset of organisational culture and is the observable degree of effort with which all the organisation members exert their attention and action towards safety. People neither deterministically controlled by their environments nor entirely self-determining. They are in a state of reciprocal determinism with their settings, where they and their environments influence one another. Safety leadership can positively impact an individual's safety-related behaviour by up to $86 \%$ and reduce accidents by around 35\%. Evidence directs us towards safety culture and safety leadership as prominent precursors to workers' safety behaviour (WSB). This study conducts a rigorous review of approximately 20-25 published papers from 2000 to 2019 , related articles in books and articles published in the corresponding field journals. The research evidence shows that Cooper's reciprocal model of safety culture encompassing psychological, behavioural and situational factors is well supported by most studies. They offer non- existent to a weak relationship between psychological factors and strong and steady situational and behavioural factors with the safety outcomes. Organisations should concentrate $80 \%$ of their safety culture improvement efforts on situational and behavioural factors to prevent process safety and SIF'(Significant incidents and fatalities). Behavioural-based safety (BBS) process serves as a comprehensive tool in altering at-risk behaviour positively. BBS will help the practitioners design enhanced BBS intervention for a more sustainable and persistent impact on workers' safety behaviour (WSB). Further research should be undertaken to establish the empirical links of safety culture and safety leadership constructs with safe outcomes.
\end{abstract}

Keywords

Safety culture, safety leadership, safety performance, Behaviour-based safety.

Article Received: 10 August 2020, Revised: 25 October 2020, Accepted: 18 November 2020

\section{Introduction}

Organisations are good at managing safety happen to manage their operations well. Safety and overall operational excellence go hand in hand. Corporate safety culture is supposed to have a strong influence on overall workplace injuries and fatalities. After implementing occupational health and safety in the organisation, there has been significant improvement in workplace safety. However, many cases of the accident are being reported even after best of organisations management, training, strict safety rules \& regulations and best of equipment in place. The complexity of industrial systems poses a challenge for industrial safety as it can a source of deviations from normal behaviour. Traditional methods of incident investigation focus primarily on the technical aspect. But the conventional model of risk analysis is not found suitable for complex industrial setup as they call for a necessity to take into account the interactions

between human, technical and organisational components.

Evidence directs us towards safety culture and safety leadership as prominent precursors to workers' safety behaviour (WSB).

\section{Methodology}

This paper follows a systematic literature review methodology to identify, analyse and draw inference from the available literature to identify the most important antecedents of workplace safety. The study conducts a rigorous review of approximately 20-25 published papers from 2000 to 2019, related articles in books and articles published in the corresponding field journals. Evidence directs us towards safety culture and safety leadership as prominent precursors to workers' safety behaviour (WSB).

\section{Result}

\section{(i) Safety Culture}

During the 1980s, there was no empirical evidence discovered in the construct. Many process safety catastrophes resulting in hundreds and thousands of industrial workers are killed or injured every week directed to an unknown, unidentified underlying contributor if identified, could have helped improve the situation. The chain of such events across different industries the safety construct was born and explained the unknown. The term 'safety culture' first made its appearance in 1987 after the 1986 Chernobyl disaster in the OECD Nuclear agency report (INSAG, 1988) on the 1986 Chernobyl disaster.

The study reveals that safety culture at different levels of the organisation influence different types of unsafe behaviours, which the risk of work injuries in turn. Thus, it is desirable to study safety culture at different/various analytical levels (i.e., the national, sectorial and organisational) to understand the effect of culture on safety thoroughly. Safety culture is defined as the share of an organisation's culture related to safety [12]. It is the observable degree of efforts with which all the organisation members direct their efforts and actions towards improving safety. Safety culture also refers to a corporate atmosphere which influences people's 
management of safety in an organisation - the number of culture definition advocate that one definition does not fit all industries. The crust of safety culture construct is about thinking positively about safety, proactively managing safety, and behaving safely.

The results show that when the safety culture was strong, leaders' behaviour created a higher/improved safety climate among the members, which predicted the workers' perceived safety behaviours. Safety culture moderated the influence that leaders exercise on safety climate. Cooper's (2000) Reciprocal safety culture Model (Fig. 1) encompassing psychological factor (safety climate), behavioural (safety behaviour) and situational factors (safety management system) has been supported by most of the academicians and researchers working on accident prevention and safety enhancement [7].

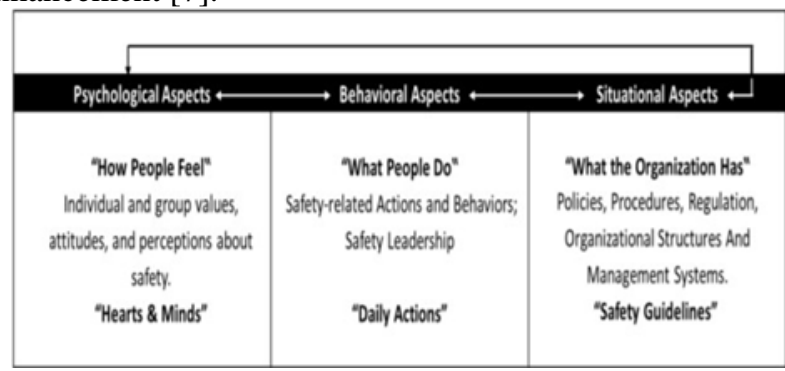

Fig 1: Cooper (2000): Reciprocal Safety Culture Model

People are neither deterministically controlled by their environments nor completely self-determining [12]. They are in a state of reciprocal determinism. They and their environments influence each other. 'Bandura's model of reciprocal determinism' offers a perfect framework to measure and analyse 'safety culture' by a triangular methodology to perform multi-level analyses. This triangulation lets the researcher take a multi-faceted understanding of safety culture. Organisations can study the reciprocal relationship between situational, psychological, and behavioural factors with an opinion to establish antecedents- behaviour-consequence path. As the elements are measurable, it is possible to quantify the 'safety culture' in a more meaningful way at many different organisational and analytical levels. The reciprocal framework provides a suitable means by which the prevailing safety culture of other business units, departments or work areas can be measured and compared [12]. Previous research findings suggest that change initiatives that disregard this interactive relationship while developing a safety culture are bound to failure.

Cooper's (2002) business model of safety culture (Fig.

2) treats the psychological ('hearts \& minds'), behavioural ('daily actions') and situational ('Safety guidelines/systems') aspects as the input to the safety culture construct which undergo a transformational process via leadership through organisational goals, expectations and management practices to produce/form the safety culture. This approach has been officially accepted by the American Petroleum Association (2016) and has also been considered standard by the American National Standards Institute (ANSI).

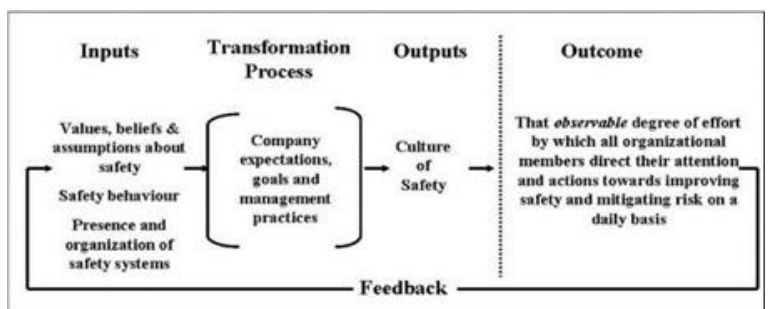

Fig 2: Cooper (2002): Business Process Model of Safety Culture

Defining 'safety culture' construct as a product helps us with a means to measure the degree of existing safety culture in the organisation. The collective evidence depicts minimal to no relationship of psychological aspects with safety outcome. It also means that the use of psychological factors as a substitute for safety culture is almost incorrect. Change and modifying situational aspects by introducing an efficient safety management system (SMS) that codifies and directs individuals' behaviour significantly optimises the safety conditions responsible for process safety accidents and personal injuries. Finally, there is a large body of work, primarily aimed at improving workers safety behaviour (WSB) in a range of industries and countries known as "Behavioural-Based Safety" (BBS). This is an approach aimed at improving workplace safety by modifying unsafe behaviour to safe behaviour.

\section{(ii) Leadership}

Leadership is defined as the capacity to influence people through personal attributes and behaviour to achieve a common goal and an essential element in guaranteeing organisations' safe running. Effective leaders understand that health and safety are not just a moral responsibility, but also contribute to achieve the organisational objectives across finance, operations, compliance and governance.

A manager's leadership style and visual demonstration of their commitment to overall health \& safety through their actions play a crucial role in shaping safety culture. The present study aims to find out how leader behaviours influence employees' safety behaviours (perceived safety behaviours). To answer this question, we have considered how this influence is exercised, taking into consideration some essential factors like safety performance, safety compliance \& safety participation. The analyses revealed that transformational leadership is characterised leaders behaviour that transforms and inspire followers to go above and beyond their mere self-interest, promote supervisory safety practices, create a positive, supportive environment, better safety behaviours and fewer accidents. At the same time, transactional leaders are just concerned with safety performance through constructive and corrective measures. In other words, transactional safety leaders promote safety performance (i.e. use of PPE \& safety compliance) and transformational safety leaders encourage "safety participation". Several studies revealed the co- occurrence of transformational and transactional safety leadership and claim that while safety leaders need transactional skills but in the absence of transformational skills it is impossible to generate employee's engagement towards health \& safety. From the past few years "safety participation" has become 
the focus of much recent behavioural safety research. Initially, various acts of employees' safety participation were presented as a single construct, implying that all actions were of equal importance in

predicting organisational safety outcome. Recent studies propose safety participation behaviour in two distinct categories: prosocial and proactive safety participation behaviour and the type of effect they exert on different safety performance outcomes such as micro-accidents, property damage, lost time injury and near-miss reporting event. In recent studies, Prosocial behaviour was found to be useful in predicting the frequency of micro-accidents and accidents without injury (property damage). In contrast, proactive behaviour played a vital role in predicting the frequency of lost time injury and near- miss events (Curcuruto et al.,2015).

\section{Discussion}

\section{Behaviour-Based Safety and its role in changing Behaviour}

Research has shown that up to $80 \%$ of workplace accidents are caused by workers behaviour, so it is imperative to understand why employee behave unsafely. Pareto's law dictates that $80 \%$ of the consequences stem from $20 \%$ of the causes. Application of this principle on accidents demonstrates that $20 \%$ of behaviours are responsible for $80 \%$ of accidents. Therefore, logically it is advisable to restrict the focus on critical and current behaviour to achieve the desired result rather than overloading the workers to all safety acts to be performed onsite. This limits the dilution of focus and achieves the desired result. Peoples behavioural choices account for around $56 \%$ of all potential severe injuries and fatalities (SIF's). Workers may participate in risk-taking behaviour that might have life-altering consequences. Under everyday situations, they may have the ability and knowledge to perform the tasks, but she/he may not have the required motivation to carry it out [14]. A favourable work environment positively influences the operators' behaviour, and an unfavourable work environment negatively impacts the operator's behaviour. The purpose of BBS is to reduce the number of unwanted incidents caused either poor management controls and/or hazard present in the working environment: when they are triggered exclusively by 'unwanted behaviour' or/those triggered by an interaction between poor controls, hazards and behaviours. Preventative opportunities, therefore, stem from controlling unwanted behaviours, identifying/eliminating risks and tightening management controls. Behavioural safety helps to identify and fix issues in all of these areas. It is vital not to confuse this approach with inspections, looking at unsafe conditions instead of unsafe acts. However, behavioural techniques aimed at improving safety-

related behaviour reduce injuries if applied while meeting the pre-requisites before implementation. Various research evidence suggests that behavioural interventions aimed at improving an organisation's safety performance would be more effective if they target specific safety behaviour (prosocial or proactive) associated with these outcomes. Otherwise, they will have minimal to no effect.

\section{Conclusion}

Most of the studies explained the role of safety culture in shaping the behaviour of the employees and the role of leaders plays in transformation of safety culture leading to safer outcomes.

The available evidence illustrates safety climate (psychological factor) as an inconsistent and weak indicator of safety- related behaviour and sole use of psychological factor (safety climate

/attitudinal survey) as a substitute to safety culture is inappropriate.

Behavioural and situational factors demonstrate a strong and reliable relationship with actual safety behaviour/outcomes. Organisations should focus almost $80 \%$ of their safety culture improvement initiatives in modifying the situational factor by developing a robust safety system to influence and optimise safety-related behaviour to avoid process safety and SIF's. In turn, to decrease any cognitive disagreement (Festinger, 1957), people will by default adjust their way of thinking to decrease any discomfort between the way they think and the way they act .

Most of safety issues/concerns reveal that it is the management behaviours from where $80 \%$ of personal injury or process safety issues arise.

Organisations must concentrate on the behaviour of workers and management but should recognise that behavioural targets for each will be significantly different.

Behavioural-based safety interventions have proved to be successful across various industries through their exclusive bottom-up approach to manage health and safety at the workplace. There lies a scope of research into its effectiveness.

Behavioural-based techniques work best when physical environment and workplace are well maintained, and all procedures are in place. The implementation of BBS provides an opportunity to the workforce to

proactively and continuously co-operate to improve safety and health.

Supervisors safety practices (i.e., Frequent safety-specific interaction with workers) can lead to improvement in workers safety behaviour (WSB) and an improved safety climate.

A more detailed study to explore empirical links of safety culture and safety leadership constructs with safe outcomes needs be carried out.

\section{References}

[1] A. Parand and P. Foster, "BehaviouralBased Safety in the Minerals Industry: A Research Based Methodology Carried out in the UK Quarrying Sector", In Queensland Resources Council Mining Health \& Safety Conference2006.

[2] C. Pilbeam, R. Davidson, N. Doherty and D. Denyer, "Safety leaders: who are they? What do they do",2016. 
[3] D. Cooper, "Safety culture", Professional safety, 47(6), 30-36,2002.

[4] D. Zohar \& G. Luria, "The use of supervisory practices as leverage to improve safety behavior: A cross-level intervention model", Journal of safety research, 34(5), 567-577,2003

[5] D. Zohar, "Modifying supervisory practices to improve subunit safety: a leadership-based intervention model" Journal of Applied psychology, 87(1), 156,2002 .

[6] D.C.C.C. Psychol, "Navigating the Safety Culture Construct: A Review of the Evidence",2016.

[7] J. Lund and L.E. Aarø, "Accident prevention. Presentation of a model placing emphasis on human, structural and cultural factors", Safety science, 42(4), 271-324,2004.

[8] J. Mullen, E.K. Kelloway and M. Teed , "Inconsistent style of leadership as a predictor of safety behaviour", Work \& Stress, 25(1), 41-54,2011.

[9] M. Curcuruto, S.M. Conchie, M.G. Mariani, and F.S. Violante, "The role of prosocial and proactive safety behaviors in predicting safety performance", Safety science, 80, 317- 323,2015.

[10] M. Martínez-Córcoles, F. Gracia,I. Tomas and J.M. Peiró, "Leadership and employees' perceived safety behaviours in a nuclear power plant: A structural equation model" Safety science, 49(8-9), 1118-1129,2011.

[11] M. S. Abdullah, Y.H. Othman, A. Osman, \& S. N. Salahudin, "Safety culture behaviour in electronics manufacturing sector (EMS) in Malaysia: The case of flextronics", Procedia economics and finance, 35, 454-461, 2016

[12] M.D. Cooper, "Towards a model of safety culture", Safety science, 36(2), 111136,2000 .

[13] N. Gravina, B. Cummins and J. Austin, 'Leadership's role in process safety: An understanding of Behavioral science among managers and executives is needed", Journal of Organizational 316-331,2017.

Behavior Management, 37(3-4),

[14] R. Aulin, A. Ek and C. Edling, "Underlying Causes for Risk Taking Behaviour Among Construction Workers" In 10th Nordic Conference on Construction Economics and Organization (Emerald Reach Proceedings Series, Volume 2). Emerald Publishing Limited (pp. 419-426),2019.

[15] S. Zhang, X. Shi and C. Wu, "Measuring the effects of external factor on leadership safety behavior: Case study of mine enterprises in China", Safety science, 93, 241-255,2017 